\title{
Uso del plasma rico en plaquetas en cirugía ligamentosa de rodilla
}

\author{
DAVID FIGUEROA P. ${ }^{1}$, FRANCISCO FIGUEROA B. ${ }^{\mathrm{a}}$, \\ XIMENA AHUMADA P. ${ }^{\mathrm{a}}$, RAFAEL CALVO R. ${ }^{1}$, ALEX VAISMAN B. ${ }^{1}$
}

\section{Use of platelet rich plasma in knee ligament surgery}

Platelet Rich Plasma (PRP) is used in musculoskeletal lesion surgery, including muscle, bone, tendons and ligaments. PRP might accelerate the healing process and the integration of the graft, allowing an earlier return to sports activities of patients. $P R P$ is obtained from autologous blood, which is centrifuged, obtaining platelet and supposedly growth factor concentrations three to five times higher than those of regular blood. The clinical results of studies performed in Chile and elsewhere on $P R P$ use in knee anterior cruciate ligament (ACL) reconstruction have been variable. Therefore, there is not enough evidence to support or deny the usefulness of PRP in ACL reconstructions.

(Rev Med Chile 2013; 141: 1315-1320).

Key words: Anterior Cruciate Ligament; Intercellular signaling peptides and proteins; Platelet-Rich Plasma.

\author{
'Equipo de Cirugía de \\ Rodilla, Servicio de \\ Traumatología Clínica \\ Alemana de Santiago, \\ Chile.

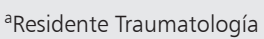 \\ Facultad de Medicina \\ Clínica Alemana- \\ Universidad del Desarrollo. \\ Recibido el 2 de septiembre \\ de 2012, aceptado el 4 de \\ diciembre de 2012. \\ Correspondencia a: \\ Dr. David Figueroa P. \\ Vitacura 5951 \\ Teléfono: 56-2-22101014. \\ E-mail: franciscofigueroab \\ @gmail.com
}

L as lesiones músculo-esqueléticas son una de las principales causa de morbilidad en todo el mundo ${ }^{1}$. En este contexto, las lesiones ligamentosas principalmente en la rodilla, las cuales están frecuentemente asociadas a la práctica deportiva, son una causa frecuente de consulta en nuestro medio, siendo la rotura del ligamento cruzado anterior (LCA) la más reconocida ya que se produce con una incidencia relativamente alta $y$ tiene el potencial de causar inestabilidad funcional al paciente, lo que puede conducir a lesiones de otros ligamentos, cartílago, meniscos y, finalmente, al desarrollo de enfermedad degenerativa de la articulación de la rodilla debido a episodios recurrentes de inestabilidad ${ }^{2-4}$. Las opciones de tratamiento deben considerar la edad del paciente, el grado de inestabilidad y el tipo e intensidad de las actividades deportivas, entre otros factores. Opciones no quirúrgicas dificultan el seguir practicando deporte, lo cual es un factor relevante en las expectativas de este tipo de pacientes.

En los últimos años, la ingeniería de tejidos y nuevas tecnologías se están introduciendo en el campo de la cirugía ortopédica con resultados aus- piciosos en diversos campos. Dentro de este grupo se encuentra el factor de crecimiento derivado de plaquetas (PDGF por sus siglas en inglés) el cual si bien se conoce desde hace más de 30 años, su uso en cirugía ortopédica data de la década de 199099, siendo en este siglo donde ha alcanzado su mayor reconocimiento. Este tendría propiedades reparadoras, estando involucrado en la mayoría de los procesos biológicos de remodelación del organismo y siendo fundamental en la regeneración de hueso y tejidos blandos ${ }^{1,5}$. Se cree que gracias a estas propiedades el PDGF podría acelerar la reparación de tendones y ligamentos ${ }^{1}$, permitiendo así un retorno precoz a la actividad deportiva previa en pacientes con lesiones músculo-esqueléticas ${ }^{6}$.

En la actualidad, el uso de PDGF es cada vez mayor en cirugía ortopédica como agente sinérgico en el tratamiento de diferentes patologías, entre ellas: reparación ósea, incluyendo la búsqueda de acelerar la consolidación de fracturas (sobre todo en pacientes con alto riesgo de no unión), fusiones de columna, tratamiento de no unión de fracturas de huesos largos, reparaciones ligamentosas e incluso el manejo de la artrosis ${ }^{6,7}$. 
En cuanto a reconstrucciones de LCA, se piensa que podría acelerar la cicatrización e incorporación del injerto utilizado en la reconstrucción y, mediante esto, permitir un retorno más rápido a las actividades de la vida diaria y deporte sin restricciones ${ }^{8}$.

Las plaquetas contienen en su interior tres tipos de compartimentos de almacenaje, cuyo contenido es liberado a la circulación al activarse. Estos son: 1. Los gránulos densos, ricos en adenosin difosfato (ADP) y serotonina; 2. Los lisosomas, que contienen enzimas proteolíticas e hidrolíticas y 3. Los gránulos alfa, los cuales poseen numerosas proteínas con gran importancia en la cicatrización, incluyendo el previamente mencionado PDGF y otros como TGF- $\beta$ (Transforming Growth Factor Beta, en inglés), PF4 (Platelet Factor 4, en inglés), interleukina 1 (IL-1), PDAF (Platelet-derived Angiogenesis Factor, en inglés) y VEGF (Vascular Endotelial Growth Factor, en inglés) entre muchos más ${ }^{6}$.

Al activarse los gránulos alfa se produce la fusión de ellos con la membrana celular de la plaqueta, proceso llamado degranulación, momento en el cual los factores de crecimiento previamente mencionados pasan a un estado de activación uniéndose a receptores transmembrana de distintos grupos celulares (stem cells mesenquimáticas, osteoblastos, fibroblastos, etc.). Esto produce expresión de genes que llevan a proliferación celular y formación de distintos tejidos 9 .

El proceso de degranulación se inicia dentro de 10 min luego de la formación del coágulo por las plaquetas y $95 \%$ de los factores de crecimiento son liberados dentro de la primera hora. Las plaquetas se mantienen por 5 a 10 días, siendo reemplazadas por macrófagos (llamados a la zona lesionada por estímulo de los factores liberados por las plaquetas) los cuales continúan con la liberación de factores de crecimiento y toman el relevo en el proceso de cicatrización?.

Basándose en este concepto se han desarrollado múltiples técnicas, que mediante la centrifugación de sangre autóloga, obtienen un concentrado con cantidades de plaquetas entre 3 a 5 veces superior a la normal, aunque se reportan en distintos estudios rangos de concentración de entre 2 y 8,5 veces la concentración normal de plaquetas ${ }^{10}$. Este centrifugado es el llamado "plasma rico en plaquetas" (PRP) el cual finalmente es el compuesto utilizado en la práctica clínica y sobre el cual se basan

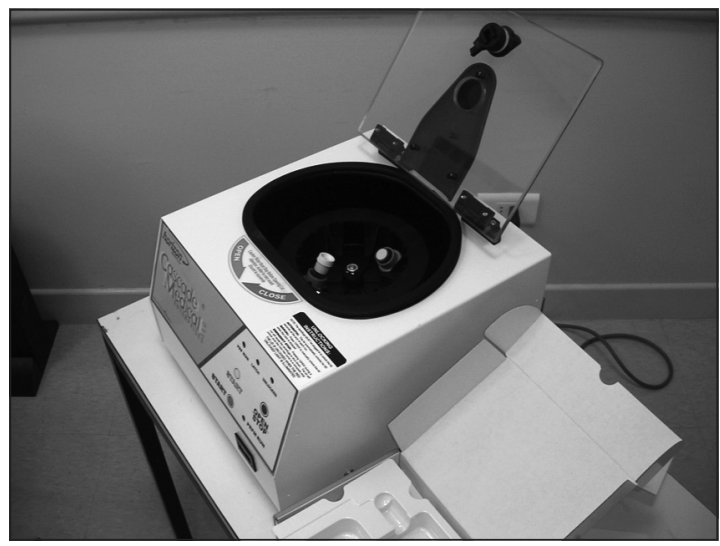

Figura 1. Máquina de centrifugado.

la mayoría de los resultados que enseñaremos a continuación.

\section{Preparación del PRP}

El mecanismo para la preparación de PRP consiste en la extracción de sangre periférica del paciente, seguido por centrifugación (Figura 1) para obtener una concentración de plaquetas muy por encima de línea basal del plasma, separadas de glóbulos rojos y leucocitos ${ }^{11}$. Es importante tener presente que hay diferencias entre los distintos sistemas comerciales con respecto al protocolo de preparación, la cantidad de sangre a extraer, el tiempo de centrifugación, el medio de transporte, el volumen final de plaquetas, el uso de anticoagulantes, la inclusión de leucocitos, y el uso de activadores que pueden dar lugar a distintos efectos biológicos ${ }^{11}$. Cuál es la cantidad óptima de plaquetas y de factores de crecimiento a utilizar en cirugía ortopédica sigue siendo tema de debate en la actualidad. Se definió como PRP con efecto clínico uno que tuviera al menos 4 veces la concentración de plaquetas normales en el plasma ${ }^{9}$, sin embargo, se han visto resultados con concentraciones menores ${ }^{12,13}$.

Todas las preparaciones de PRP tienen pasos en común. El producto se centrifuga estratificádamente en tres capas. La base o capa roja, está llena de eritrocitos, la capa media o blanca, contiene los leucocitos los cuales contienen citoquinas inflamatorias y la capa superior o amarilla, contiene plasma y plaquetas con los factores de crecimiento previamente mencionados en su interior ${ }^{14}$. Esta 
última capa será la que se administrará en la región donde se requiera.

Las principales diferencias entre los diferentes sistemas de preparación son la velocidad y el número de centrifugaciones, lo que conduce a diferentes concentraciones de plaquetas. Un sistema que ofrezca un mayor número absoluto de plaquetas no necesariamente será mejor, sino que debe atenerse a parámetros mínimos y máximos que producen un efecto óptimo en la reparación ósea, lo cual se ha demostrado que se consigue con concentraciones de plaquetas entre 503.000 y 1.729.000 plaquetas/uL de $\mathrm{PRP}^{15}$. Concentraciones por debajo de 380.000 plaquetas/uL tendrían un efecto sub-óptimo y concentraciones por encima de 1.800 .000 plaquetas/uL podrían tener un efecto paradójico en el cual inhibirían la reparación ${ }^{15}$.

Como se mencionó anteriormente, la activación de las plaquetas es necesaria para liberar factores de crecimiento de los gránulos en los cuales están contenidos. Algunos sistemas comerciales no activan los preparados, con el argumento de que estos se activarán una vez en el campo quirúrgico ${ }^{16,17}$. Otros utilizan trombina exógena o endógena inmediatamente antes de la administración (Figura 2), para así activar la cascada de degranulación de las plaquetas, la formación del coágulo y la liberación de factores de crecimiento en el sitio de inyección ${ }^{10}$. Otro activador utilizado es el cloruro de calcio, el cual no tendría el riesgo de reacciones de hipersensibilidad de la trombina exógena ${ }^{1}$. La inclusión de leucocitos dentro del PRP también es controversial. Si bien quienes la favorecen basan su argumento en la posibilidad de disminuir las tasas de infección local, se ha visto que provocaría efectos deletéreos producto de metaloproteinasas las cuales podrían provocar daño tisular importante en la zona de inoculación, razón por la cual no se incluyen de regla en los preparados ${ }^{1}$.

\section{Evidencia en su uso clínico}

Los resultados exitosos en reconstrucciones de LCA han sido variables según las distintas series, abarcando un rango de 73 a 95\% de éxito y 37 a $75 \%$ de retorno a la actividad deportiva previa sin limitaciones ${ }^{14}$. El tiempo de retorno a la actividad deportiva varía entre 6 y 12 meses debido a que la reconstrucción requiere un mínimo de tiempo para su integración al tejido del receptor de forma que este pueda realizar su actividad normal sin un alto riesgo de re-rotura ${ }^{14}$. Es en estos procesos en los cuales el aporte de PRP (Figura 3) busca acelerar y mejorar los resultados clínicos y en los cuales están enfocados la gran mayoría de los estudios en este campo. Es importante destacar que dentro de los múltiples usos del PRP (Cirugía Estética y Reconstructiva, Odontología y otros usos en Traumatología) su aplicación en la cirugía ligamentaria de rodilla es la que cuenta con mayor cantidad de estudios comparables, principalmente porque se esta tratando una aplicación en un lugar común (sitio lesionado), una lesión singular (en este caso LCA) y en un tipo similar de pacientes (jóvenes y deportistas).

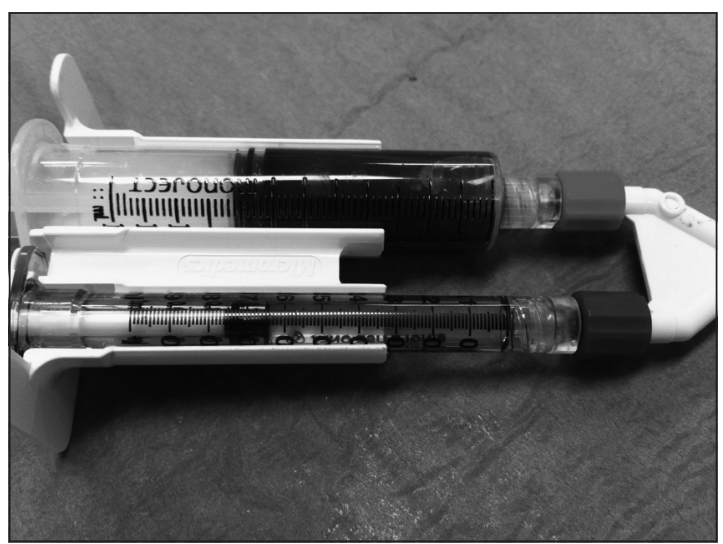

Figura 2. PRP (jeringa ancha) y Trombina (jeringa delgada).

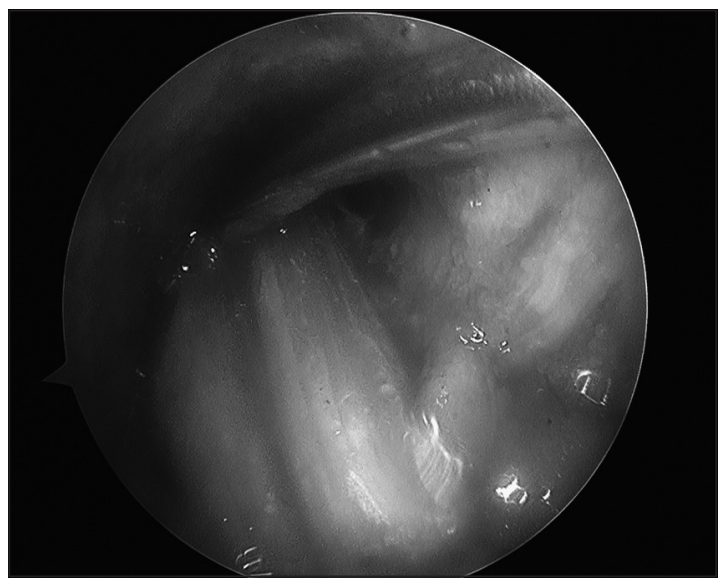

Figura 3. Adición de PRP en injerto de LCA. 
Fanelli y cols siguieron 70 reconstrucciones de LCA a los cuales se les adicionó PRP. El estudio radiográfico de estos pacientes mostró $6,7 \%$ de expansión del túnel y osteolisis de este, mientras que en aquellos que no recibieron suplementación con PRP se encontró una incidencia de $52 \%$ de estas complicaciones ${ }^{18}$. Recientemente, una revisión sistemática proveniente de la Universidad de Harvard concluyó que la evidencia actual sugiere que la adición de concentrado plaquetario a la reconstrucción de LCA podría tener un efecto benéfico en la maduración del injerto, pudiendo mejorar entre $20 \%$ a $30 \%$ en promedio este ámbito. La forma más probable de acción sería que las plaquetas aceleran la repoblación y remodelación del injerto ${ }^{19}$.

España es el país que más dedicación le ha dado a la investigación de los factores de crecimiento plaquetarios y su utilidad clínica. Los primeros estudios datan de la década 1990-99 con Sánchez como uno de sus precursores. En uno de sus estudios, comparó una cohorte de 50 pacientes tratados con reconstrucción de LCA más adición de PRP tanto en el injerto como en el sitio donante, con una cohorte de 50 pacientes sometidos a reconstrucción de LCA sin uso de PRP. Encontraron que en el grupo con PRP hubo menos hematomas postoperatorios importantes en el sitio donante y menor necesidad de nuevas intervenciones quirúrgicas por rigidez de rodilla. También hubo resultados positivos respecto a la estabilidad y mejor integración radiológica del injerto en los túneles en el primer mes después de la cirugía ${ }^{20}$. Por el contrario, Nin y cols, en un estudio prospectivo aleatorio de 100 pacientes sometidos a artroscopia y reconstrucción de LCA con aloinjerto de tendón rotuliano con y sin uso de PRP concluyen que el PRP no tiene ningún efecto discernible tanto clínico como biomecánico ${ }^{8}$.

Silva y Sampaio, en Portugal, realizaron un estudio prospectivo de 40 pacientes con reconstrucciones de LCA doble banda con autoinjerto de tendones semitendinoso y gracilis, los cuales fueron divididos en 4 grupos distintos: un primer grupo control, un segundo grupo al cual se le adicionó PRP no activado en los túneles de la reconstrucción ligamentaria, un tercer grupo al cual se le adicionó PRP activado en los túneles, y un cuarto grupo al cual se le adicionó PRP no activado en los túneles quirúrgicos y luego se le realizó infiltración con PRP no activado a la segunda y cuarta semana postoperatorias. Se realizó control con resonancia magnética (RM) a los 3 meses concluyendo que no había diferencias entre los grupos en el proceso de integración del injerto ${ }^{21}$.

En Latinoamérica, Chile es el país pionero en la utilización y estudio sobre este tema. Radice, en el año 2010, publicó un estudio prospectivo simple ciego de 50 reconstrucciones de LCA evaluando los hallazgos en la RM de los injertos sometidos a PRP en contraste con los cuales aunque no se utilizó PRP. Se concluyó que la reconstrucción de LCA con el uso de PPR logra completar injertos homogéneos en 179 días, en comparación con 369 días para la reconstrucción de LCA sin PPR. Esto representa una reducción del tiempo de maduración de $48 \%$, lo que sugiere que el PRP aportaría a la velocidad de incorporación del injerto en reparaciones de LCA y, por tanto, se plantea que esto tendría utilidad en un retorno deportivo precoz ${ }^{22}$.

Otro estudio prospectivo aleatorio (Orrego y cols.) realizado en nuestro país en, el cual se aplicó PRP a 26 pacientes de un total de 108 reconstrucciones de LCA, llegó a la conclusión de que el uso de PRP tenía un efecto sinérgico en el proceso de maduración del injerto evaluado por la intensidad de señal en la RM, sin mostrar ningún efecto significativo en la interfaz osteo-ligamentosa o en la evolución del túnel ${ }^{23}$.

Por último, nuestro grupo de trabajo el año 2010 publicó un estudio prospectivo aleatorio simple ciego que se realizó en dos series consecutivas de pacientes que se sometieron a reconstrucción de LCA en un período de 14 meses: En 30 pacientes se utilizó PRP y 20 pacientes fueron denominados como grupo control sin la adición de PRP. Se concluyó que a los 6 meses post reconstrucción, no existe un beneficio estadísticamente significativo en el grupo con PRP en relación a la integración y la maduración del injerto de LCA medido en $\mathrm{RM}^{24}$.

\section{Conclusiones}

La cirugía ligamentosa más frecuente realizada en la cirugía de rodilla es la reconstrucción de LCA, la cual se produce principalmente en pacientes sometidos a exigencias deportivas importantes y en los cuales los resultados clínicos y el tiempo de reincorporación a su nivel deportivo previo a la lesión son de altísima importancia. Es por esta ra- 
zón que se han buscado nuevas formas de acelerar el proceso de integración del injerto utilizado en la reconstrucción y además obtener resultados clínicos óptimos. Dentro de esta categoría se encuentra el plasma rico en plaquetas, el cual teóricamente debería tener la capacidad de acelerar los procesos de reparación de los tejidos mediante la liberación de factores de crecimiento. Para comprobar estas teorías se han realizado múltiples estudios, tanto en Norteamérica y Europa, como en nuestro país.

Luego de la revisión sistemática de la literatura disponible y de nuestras experiencias personales, podemos concluir que aún no existe evidencia suficiente tanto para asegurar o descartar la utilidad del PRP en las reconstrucciones ligamentarias de rodilla. Los resultados entre los distintos estudios son dispares y existe actualmente una falta evidencia nivel I que avale su uso. Finalmente, la mayoría de los estudios se enfoca en parámetros radiológicos, sin embargo, es necesario realizar estudios clínicos que demuestren si efectivamente los pacientes logran una recuperación más rápida y, por tanto, un retorno precoz a su actividad deportiva previa.

\section{Referencias}

1. Anitua E, Sánchez M, Nurden AT, Nurden P, Orive G, Andía I. New insights into and novel applications for platelet-rich fibrin therapies. Trends Biotechnol 2006; 24 (5): 227-34.

2. Lohmander LS, Englund PM, Dahl LL, Roos EM. The long-term consequence of anterior cruciate ligament and meniscus injuries: osteoarthritis. Am J Sports Med 2007; 35: 1756-69.

3. Øiestad BE, Engebretsen L, Storheim K, Risberg MA. Knee osteoarthritis after anterior cruciate ligament injury: a systematic review. Am J Sports Med 2009; 37: 1434-43.

4. Fithian DC, Paxton LW, Goltz DH. Fate of the anterior cruciate ligament injured knee. Orthop Clin North Am 2002; 33: 621-36.

5. Anitua E, Andía I, Ardanza B, Nurden P, Nurden AT. Autologous platelets as a source of proteins for healing and tissue regeneration. Thromb Haemost 2004; 91: 4-15.

6. Anitua E, Sánchez M, Orive G, Andía I. The potential impact of the preparation rich in growth factors (PRGF) in different medical fields. Biomaterials 2007; 28: 455160 .

7. Eppley BL, Pietrzak WS, Blanton M. Platelet-rich plasma: A review of biology and applications in plastic surgery. Plast Reconstr Surg 2006; 118: 147-59.

8. Nin JR, Gasque GM, Azcárate AV, Beola JD, González $\mathrm{MH}$. Has platelet-rich plasma any role in anterior cruciate ligament allograft healing? Arthroscopy 2009; 25 (11): 1206-13.

9. Marx RE. Platelet-rich plasma: Evidence to support its use. J Oral Maxillofac Surg 2004; 62: 489-96.

10. Kevy SV, Jacobson MS. Comparison of methods for point of care preparation of autologous platelet gel. J Extra Corpor Technol 2004; 36: 28-35.

11. Hall MP, Band PA, Meislin RJ, Jazrawi LM, Cardone DA. Platelet-rich plasma: current concepts and application in sports medicine. J Am Acad Orthop Surg 2009; 17 (10): 602-8.

12. Eppley BL, Woodell JE, Higgins J. Platelet quantification and growth factor analysis from platelet-rich plasma: Implications for wound healing. Plast Reconstr Surg 2004; 114: 1502-8.

13. Sánchez M, Anitua E, Azofra J, Andía I, Padilla S, Mujika I. Comparison of surgically repaired Achilles tendon tears using platelet-rich fibrin matrices. Am J Sports Med 2007; 35: 245-51.

14. López-Vidriero E, Goulding KA, Simon DA, Sánchez $\mathrm{M}$, Johnson DH. The use of platelet-rich plasma in arthroscopy and sports medicine: optimizing the healing environment Arthroscopy 2010; 26 (2): 269-78.

15. Weibrich G, Hansen T, Kleis W, Buch R, Hitzler WE. Effect of platelet concentration in platelet-rich plasma on peri- implant bone regeneration. Bone 2004; 34 : 665-71.

16. Mishra A, Pavelko T. Treatment of chronic elbow tendinosis with buffered platelet rich plasma. Am J Sports Med 2006; 34: 1774-8.

17. Fufa D, Shealy B, Jacobson M, Kevy S, Murray MM. Activation of platelet-rich plasma using soluble type I collagen. J Oral Maxillofac Surg 2008; 66: 684-90.

18. Arnoczky SP, Anderson L, Fanelli G, Ho S, Mishra A, Sgaglione N. The role of platelet-rich plasma in connective tissue repair. Orthopedics Today 2009; 26: 29.

19. Vavken P, Sadoghi P, Murray MM. The effect of platelet concentrates on graft maturation and graft-bone interface healing in anterior cruciate ligament reconstruction in human patients: a systematic review of controlled trials. Arthroscopy 2011; 27 (11): 1573-83.

20. Sánchez M, Azofra J, Aizpurua B, Elorriaga R, Anitua E, Andia I. Use of autologous plasma rich in growth factors in arthroscopic surgery. Cuad Artrosc 2003; 10: 12-9.

21. Silva A, Sampaio R. Anatomic ACL reconstruction: Does the platelet-rich plasma accelerate tendon healing? Knee Surg Sports Traumatol Arthrosc 2009; 17: 676-82.

22. Radice F, Yánez R, Gutiérrez V, Rosales J, Pinedo M, 
Coda S. Comparison of magnetic resonance imaging findings in anterior cruciate ligament grafts with and without autologous platelet-derived growth factors. Arthroscopy 2010; 26 (1): 50-7.

23. Orrego M, Larraín C, Rosales J, Valenzuela L, Matas J, Durruty J et al. Effects of platelet concentrate and a bone plug on the healing of hamstring tendons in a bone tunnel. Arthroscopy 2008; 24 (12): 1373-80.

24. Figueroa D, Melean P, Calvo R, Vaisman A, Zilleruelo N, Figueroa $\mathrm{F}$, et al. Magnetic resonance imaging evaluation of the integration and maturation of semitendinosusgracilis graft in anterior cruciate ligament reconstruction using autologous platelet concentrate. Arthroscopy 2010; 26 (10): 1318-25. 